\title{
An Overview of Cooperative Driving in the European Union: Policies and Practices
}

\author{
Marilisa Botte $^{1, *(\mathbb{D}}$, Luigi Pariota ${ }^{1,2} \mathbb{(}$, Luca $\mathrm{D}^{\prime}$ Acierno $^{1}{ }^{1}$ and Gennaro Nicola Bifulco ${ }^{1}(\mathbb{C}$ \\ 1 Department of Civil, Architectural and Environmental Engineering, Federico II University of Naples, Via \\ Claudio 21, 80125 Naples, Italy; luigi.pariota@unina.it (L.P.); luca.dacierno@unina.it (L.D.); \\ gennaro.bifulco@unina.it (G.N.B.) \\ 2 LAERTE-ITS - Laboratory for Advanced Experiments on Roads and Traffic Environments, Federico II \\ University of Naples, Corso Nicolangelo Protopisani 70, 80146 Naples, Italy \\ * Correspondence: marilisa.botte@unina.it; Tel.: +39-081-768-3356
}

Received: 17 April 2019; Accepted: 28 May 2019; Published: 31 May 2019

\begin{abstract}
Cooperative-Intelligent Transportation Systems (C-ITSs) aim to connect vehicles, both with one another and with road infrastructures, so as to increase traffic safety and efficiency. This paper focuses on the European framework for supporting the development of Cooperative, Connected, and Automated Mobility, and aims to shed light on the current state of testing and deployment activities in the field at the start of 2019. This may be considered particularly timely given that the year 2019 was identified as the starting date for the deployment of mature services, and the Community legislation is currently paying great attention to the matter. In order to present a concise (but comprehensive) picture, we consulted and analysed the most diverse sources comprising more than 2000 pages.
\end{abstract}

Keywords: cooperative driving; European framework; smart roads; C-ITS services; open-road pilot sites; vehicle to everything (V2X) testbeds

\section{Introduction}

Cooperative-Intelligent Transportation Systems (C-ITSs) represent the set of technological and functional elements that allow specific communication tasks identified as V2X (i.e., vehicle to everything) communication services. The ' $X$ ' in $\mathrm{V} 2 \mathrm{X}$ can identify another vehicle (i.e., $\mathrm{V} 2 \mathrm{~V}$ communication) or the infrastructure (i.e., V2I communication). In some approaches, ' $X$ ' is also used to identify the cloud. The primary goal of such technologies is to improve road safety by helping the driver make the right decision and adapt to traffic conditions, thereby avoiding potential harm. This clearly means abating road fatalities and injury severity. Under the perspective of a progressively growing level of driving automation, these technologies could be directly exploited by the vehicle without the intervention of the driver. Other potential benefits of the use of C-ITS include enhanced traffic efficiency and improved driving experience. Indeed, the use of V2X technologies is assumed to reduce congestion and make driving tasks less reliant on human action. Finally, they can ensure environment-friendly driving through in-vehicle technologies (e.g., eco-driving), and smarter transportation management at the network level. As stated above, communication and cooperation among vehicles and between vehicles and infrastructure are crucial for the safe integration and operation of automated vehicles in transport systems of the future.

Within this framework, both the vehicles and the infrastructures have to be smart agents, and a reliable communication channel between them needs to be built. Therefore, C-ITS development has two main levers: enrichment of in-vehicle technologies and infrastructural development. Other crucial elements concern regulations, service standardisation, and cyber security issues. 
Community legislation is paying great attention to the matter. In March 2019, a delegated act supplementing Directive 2010/40/EU was released [1]. Specifically, this new regulation concerning Cooperative-Intelligent Transport Systems is planned to enter into force and be directly applicable in all Member States from 31 December 2019. In light of the above, this paper aims to provide a concise (but comprehensive) overview of the current state of C-ITS testing and development activities across the European Union (EU). The challenge is to collect, examine, and summarise the countless sources available in their most diverse forms. Indeed, not only have official documents describing standards and project reports been consulted, but press releases, online articles, and datasheets have also been examined, amounting to more than 2000 pages. The goal consists in identifying C-ITS definitions, implemented technologies, level of readiness of different kinds of services (i.e., some of them are ready to be deployed while others require additional research and experimental phases), operating consortia, infrastructural development and other relevant factors, and presenting them in a synoptic and straightforward way. Clearly, although our review pays particular attention to the European case, it is worth noting that similar phenomena are occurring in the USA, Australia, and in the Far East.

The remainder of the paper is organised as follows: Section 2 describes the key features of the C-ITS European framework; Section 3 illustrates the basic principles of cooperative services and technologies; Section 4 outlines the best practices across the EU; finally, Section 5 provides the concluding remarks, including lessons learned and future perspectives.

\section{The European Framework}

The European Commission (EC) is making significant efforts in the field of C-ITS services, based on V2X communication, in order to set up a reference framework for supporting Cooperative, Connected, and Automated Mobility (CCAM) policies [2]. The matter is quite complex since there are several implications to be considered. Figure 1 outlines the EU framework for supporting C-ITS service development. First, C-ITS strategy aims to develop a common vision throughout the EU so as to combine the efforts of the different stakeholders involved. For this purpose, in early 2014 the EC set up the C-ITS Platform, conceived as a cooperative framework to develop a shared vision for the interoperable deployment of C-ITS in the EU. Later, in 2016, Member States and the Commission launched the C-Roads Platform to link and coordinate C-ITS deployment across the Union. Finally, on completion of the picture, there are some issues to be addressed to ensure legal certainty for the parties as well as cyber security and data privacy matters.

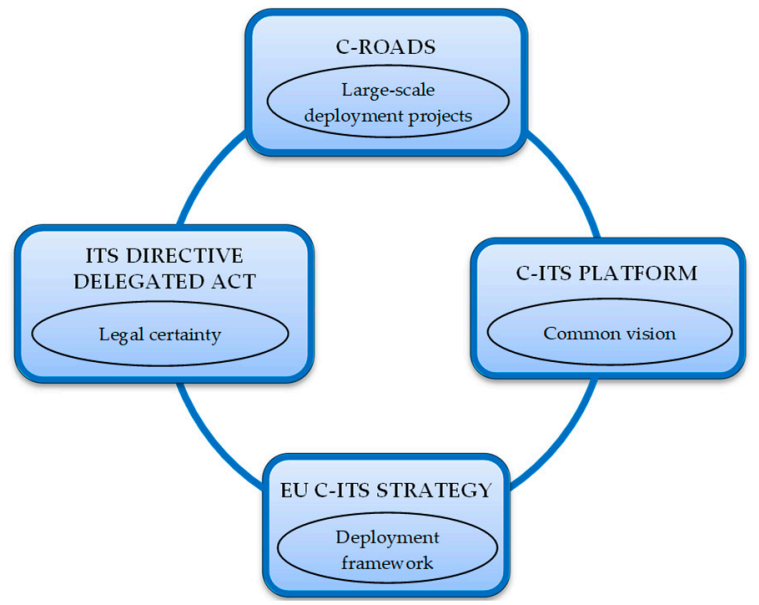

Figure 1. EU Cooperative-Intelligent Transportation Systems (C-ITS) framework.

Within the European initiative for the promotion of C-ITS, a key role is being played by the Car2Car Communication Consortium (C2C-CC) [3] and the Amsterdam Group (AG) [4]. 
C2C-CC it was founded in 2002 by a group of carmakers and today comprises 88 members among carmakers, Original Equipment Manufacturers (OEMs), first- and second-tier suppliers, technology suppliers, and research organisations. $\mathrm{C} 2 \mathrm{C}-\mathrm{CC}$, in turn, is part of the Amsterdam Group (AG). In particular, AG is a strategic alliance formed in 2011 to support close cooperation among the stakeholders involved. Beyond C2C-CC, it comprises three other umbrella organisations: CEDR (European organisation for national road administrations), ASECAP (European association of toll road operators), and POLIS (European cities and regions network). More details about such consortia and the roadmaps they proposed for the development of cooperative driving can be found in [5].

However, it is worth pointing out that the Society of Automotive Engineers (SAE) has established a similar evolution pattern, based on different levels of increasing automation (from Level 1 to Level 5), specifically developed for autonomous driving [6]. Clearly, both for cooperative and autonomous driving, the final goal is to achieve a fully automated condition. Yet, specific correspondence between the evolution phases in the two cases has not been established, and the position of the Commission on this matter has been expressed as follows: 'even though automated vehicles do not necessarily need to be connected and connected vehicles do not require automation, it is expected that, in the medium term, connectivity will be a major enabler for driverless vehicles' [7].

To complete the picture, it is worth citing the extensive consultation process undertaken by the Commission through several working groups called upon to analyse the current situation and provide useful recommendations for the future. An appropriate instance of this is the establishment of the High Level Group (HLG) Gear 2030 in 2015. The outcome of such a consultation process is presented in [8] and provides some valuable suggestions to address the main challenges and opportunities offered by cooperative driving in the runup to 2030 and beyond.

\section{Cooperative-Intelligent Transportation Systems (C-ITS) Services and Technologies Adopted in the European Commission (EC) Framework}

C-ITS deployment activities have also focused on technological and functional aspects of the matter, which essentially concern the definition of a number of so-called C-ITS services and a set of reliable communication technologies.

A first macrocategory of cooperative tasks comprises hazardous location notifications (HLNs), which are intrinsically safety-critical services. In particular, they are aimed at providing an early warning to drivers about dangerous conditions ahead. Among them, we can find tasks for warning drivers of hard braking by vehicles ahead (i.e., emergency electronic brake light-EBL) as well as for providing an early warning of approaching emergency vehicles (i.e., emergency vehicle approaching-EVA) prior to the siren or light bar being audible or visible. Moreover, slow or stationary Vehicle (SSV) service is designed for warning approaching drivers about slow or stationary/broken down vehicles ahead, while traffic jam ahead warning (TJW) has the purpose of providing an alert to the driver on approaching the tail end of a traffic jam. This can be particularly useful when, for example, the tail end is hidden behind a hilltop or curve. Other typical HLN tasks are those concerning road works warning (RWW) and weather conditions (WTCs) services, which are aimed, respectively, at informing drivers about current road works and providing accurate and current local weather information. This is particularly useful in the case of dangerous weather conditions, which are difficult to perceive visually, such as black ice or strong gusts of wind. Additional cooperative services belonging to the HLN group are: cooperative collision risk warning (CCRW), whose purpose is minimising the risk of collision during overtaking or merging with traffic, and motorcycle approaching indication (MCA), which is finalised to warn drivers of motorcycles arriving. Finally, the wrong-way driving (WWD) service has been designed to provide an advance warning of wrong-way driving, thus alerting, on the one hand, drivers that they are driving in the wrong direction and, on the other, warning surrounding vehicles of the danger.

By moving towards the signage applications category we can find in-vehicle signage (VSGN), which is aimed at providing advance information about relevant road signs in the vehicle surroundings to increase driver awareness, and in-vehicle speed limits (VSPDs), whose function is to inform drivers 
about speed limits continuously or on a specific occurrence. Additionally, probe vehicle data (PVD) service is aimed at collecting vehicle data for a variety of purposes concerning circulation safety and efficiency, traffic management, and environmental issues. While, for reducing the occurrence of traffic shockwaves (i.e., transition zones between two traffic states that move through a traffic environment), thus making traffic flow smoother and reducing vehicle emissions, the shockwave damping (SWD) service has been developed. Moreover, green light optimal speed advisory (GLOSA) tasks are finalised to provide speed advice to drivers approaching traffic lights, thus reducing the number of sudden acceleration or braking incidents. In particular, for PVD, SWD, and GLOSA services, the field of application is an urban environment. Other two services belonging to signage applications are signal violation/intersection safety (SigV), which is a safety-critical task and aims at reducing the number and severity of collisions at signalised intersections, and traffic signal priority request by designated vehicles (TSP), which is aimed at allowing drivers of priority vehicles (e.g., emergency vehicles, public transport, and heavy goods vehicles (HGVs)) to be given priority at signalised junctions.

Other C-ITS services are those based on infotainment tasks whose purpose is providing different kinds of information to drivers, such as off-street and on-street parking availability, alternative routes, and location of charging stations. Further, typical services developed for urban applications are: loading zone management (LZM), which is conceived for supporting the management of urban parking zones specific to freight vehicles, with benefits for drivers, fleet managers and road operators; zone access control for urban areas (ZAC), which is targeted to provide information about access restrictions to specific urban areas, thus allowing drivers to better plan their trip; and vulnerable road user protection (VRU), which is a safety-critical service to the benefit of vulnerable road users, i.e., pedestrians and cyclists. Finally, it is worth citing connected and cooperative navigation $(\mathrm{CCN})$, which represents an advanced C-ITS service for which no definitive descriptions have been released yet. However, it is based on a systemic framework in which tasks like the first and last mile, parking information, route advice, and coordinated traffic lights are fully synchronised and integrated.

A more detailed description of the features and applications of the above-mentioned C-ITS services can be found in [9-16].

Obviously, each of the services described needs to be supported by standardised messages and, as mentioned above, by suitable communication technologies. The European Telecommunications Standards Institute (ETSI) has defined two different kinds of messages, namely cooperative awareness messages (CAMs) [17] and decentralized environmental notification messages (DENMs) [18], with related packet formats and dissemination guidelines. Specifically, CAMs are a kind of heartbeat message periodically broadcast by each vehicle to its neighbours to provide information on presence, position, speed, temperature, and basic status. By contrast, DENMs are event-triggered messages broadcast to warn road users about a hazardous event. Both CAMs and DENMs are delivered to vehicles in a particular geographic region: in the immediate neighbourhood in the case of CAMs (single hop) and in the area affected by the event for DENMs (multihop) [19]. Two other kinds of standardised messages, generally disseminated by the infrastructure, are signal phase and timing (SPaT) and map data $(M A P)$ [20-22]. The former concerns the status of traffic lights, prediction of duration and phases, data elements for prioritisation response, as well as permission linked to manoeuvres or to lanes; the latter provides information about road topology (such as topological definition of lanes for a road segment or within an intersection, links between the segments, and restrictions in lanes). To complete the picture, it is worth citing in-vehicle information (IVI) messages $[23,24]$, which transmit to the vehicle static and dynamic data about infrastructure such as recommendations dictated by road signs and speed limit information.

Further, V2X data exchange can occur by means of different kinds of technologies. The most mature communication technology is described by the standard ETSI ITS G5 [25]. It is a European set of protocols and parameters for short-range communication between vehicles and traffic infrastructure, which operates in the $5.9 \mathrm{GHz}$ frequency band; not coincidentally, it is also known as dedicated short-range communication (DSRC). It is based on the IEEE standard 802.11 [26] (used for Wi-Fi) and 
its amendment 802.11p on wireless access in vehicular environments [27], which introduces several modifications to adapt the physical (PHY) layer and medium access control (MAC) sublayer to the requirements of highly dynamic vehicular environments. More details on this technology can be found in [28].

Up and coming alternative technologies consist of cellular communications; however, this technology cannot ensure a direct link since it needs to rely on the presence of the cloud and, hence, is not suitable for use in the case of safety-critical applications [29]. Nevertheless, in this regard, clarification is due. Strictly speaking, this holds for $3 G$ and $4 G$ networks. Yet, with the advent of LTE (long term evolution) and fifth generation cellular communication 5G, cutting edge technologies, defined as C-V2X (cellular-V2X), have been developed. Such innovative technologies are known as LTE-V2X (or car-LTE) [30] and 5G NR-V2X (new radio-V2X) [31]. According to their advocates, they can operate equivalently to DSRC, in ITS bands and independent of cellular networks, thus ensuring a direct channel between the interlocutors involved with a very low latency [32,33]. An extensive description of LTE-V2X communication and its evolution towards fifth generation technologies can be found in [34-36]. Finally, [37-39] provide a comparative discussion about features and applications of the above communication systems together with the identification of the related optimal application ranges of distance and safety requirements.

The European Commission set in 2019 the start time of deployment of mature C-ITS services and identifies, in the C-ITS Platform Phase II Report [40], a list of services to be considered as overriding, given their high safety potential. On the basis of the priority required for their deployment, services are classified as Day-1 and Day-1.5; however, the report specifies that the above list should not be seen as the 'official C-ITS service list' but only as a first benchmark for making the various deployment activities interoperable across the EU.

Table 1 represents an excerpt from Annex 1 to the C-ITS Platform Report [40] and shows a cooperative services classification with reference to:

- $\quad$ interlocutors involved (i.e., V2V and V2I)

- $\quad$ kind of standardised messages implemented, (i.e., CAM, DENM, SPaT, MAP, and IVI)

- communication technology adopted (i.e., ETSI G5 and traditional cellular networks)

- $\quad$ application field (i.e., urban environment and motorway)

- $\quad$ safety-related features (i.e., safety-critical (SC) and nonsafety-critical (nSC))

- $\quad$ priority in deployment (i.e., Day-1 and Day-1.5) 
Table 1. Classification of C-ITS services (source: [40]).

\begin{tabular}{|c|c|c|c|c|c|c|c|c|c|c|c|c|c|c|c|}
\hline \multirow{2}{*}{\multicolumn{2}{|c|}{$\begin{array}{c}\text { C-ITS } \\
\text { Services } \\
\end{array}$}} & \multicolumn{2}{|c|}{ Interlocutors Involved } & \multicolumn{5}{|c|}{ Standardised Messages } & \multicolumn{2}{|c|}{ Communication Technologies } & \multicolumn{2}{|c|}{ Application Field } & \multicolumn{2}{|c|}{ Safety } & \multirow{2}{*}{ Priority } \\
\hline & & V2I & V2V & CAM & DENM & SPaT & MAP & IVI & ETSI-G5 & Cellular (3G/4G) & Urban & Motorway & SC & nSC & \\
\hline \multirow{9}{*}{ 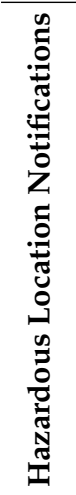 } & EBL & & $x$ & & $\mathrm{x}$ & & & & $\mathrm{x}$ & & $\mathbf{x}$ & $\mathbf{x}$ & $\mathbf{x}$ & & 1 \\
\hline & EVA & & $\mathbf{x}$ & $\mathbf{x}$ & $\mathbf{x}$ & & & & $\mathbf{x}$ & & $\mathbf{x}$ & $\mathbf{x}$ & $\mathbf{x}$ & & 1 \\
\hline & SSV & & $\mathbf{x}$ & $\mathbf{x}$ & $\mathbf{x}$ & & & & $\mathbf{x}$ & & $\mathbf{x}$ & $\mathbf{x}$ & $\mathbf{x}$ & & 1 \\
\hline & TJW & & $\mathbf{x}$ & & $\mathbf{x}$ & & & & & $\mathbf{x}$ & & $\mathbf{x}$ & $\mathbf{x}$ & & 1 \\
\hline & RWW & $\mathbf{x}$ & & & $\mathbf{x}$ & & & & & $\mathbf{x}$ & $\mathbf{x}$ & $\mathbf{x}$ & $\mathbf{x}$ & & 1 \\
\hline & WTC & $\mathbf{x}$ & $\mathbf{x}$ & & $\mathbf{x}$ & & & & & $\mathbf{x}$ & $\mathbf{x}$ & $\mathbf{x}$ & $\mathbf{x}$ & & 1 \\
\hline & CCRW & $\mathbf{x}$ & $\mathbf{x}$ & $\mathbf{x}$ & $\mathbf{x}$ & & & & $\mathbf{x}$ & & $\mathbf{x}$ & $\mathbf{x}$ & $\mathbf{x}$ & & 1.5 \\
\hline & MCA & & $\mathbf{x}$ & $\mathbf{x}$ & & & & & $\mathbf{x}$ & & $\mathbf{x}$ & $\mathbf{x}$ & $\mathbf{x}$ & & 1.5 \\
\hline & WWD & $x$ & & & $\mathbf{x}$ & & & & $\mathbf{x}$ & & $\mathbf{x}$ & $x$ & $\mathbf{x}$ & & 1.5 \\
\hline \multirow{7}{*}{ 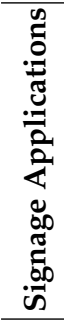 } & VSGN & $\mathbf{x}$ & & & & & & $\mathbf{x}$ & $\mathbf{x}$ & & $\mathbf{x}$ & $\mathbf{x}$ & $\mathbf{x}$ & & 1 \\
\hline & VSPD & $\mathbf{x}$ & & $\mathbf{x}$ & & & & $\mathbf{x}$ & $\mathbf{x}$ & & $\mathbf{x}$ & $x$ & $\mathbf{x}$ & & 1 \\
\hline & PVD & $\mathbf{x}$ & & $\mathbf{x}$ & & & & & $\mathbf{x}$ & & $x$ & $x$ & & $\mathbf{x}$ & 1 \\
\hline & SWD & $\mathbf{x}$ & & & & & & $\mathbf{x}$ & $\mathbf{x}$ & & & $x$ & & $\mathbf{x}$ & 1 \\
\hline & GLOSA & $\mathbf{x}$ & & $\mathbf{x}$ & & $\mathbf{x}$ & $\mathbf{x}$ & & $\mathbf{x}$ & & $\mathbf{x}$ & & & $\mathbf{x}$ & 1 \\
\hline & SigV & $\mathbf{x}$ & & & & $\mathbf{x}$ & $\mathbf{x}$ & & $\mathbf{x}$ & & $\mathbf{x}$ & & $\mathbf{x}$ & & 1 \\
\hline & TSP & $x$ & & & & $\mathbf{x}$ & $\mathbf{x}$ & & $\mathbf{x}$ & & $\mathbf{x}$ & & & $\mathbf{x}$ & 1 \\
\hline \multirow{5}{*}{ 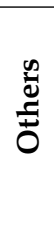 } & Info $^{1}$ & $x$ & & $x$ & & & & & & $x$ & $x$ & $\mathbf{x}$ & & $x$ & 1.5 \\
\hline & LZM & $\mathbf{x}$ & & $\mathbf{x}$ & & & & & $\mathbf{x}$ & $\mathbf{x}$ & $x$ & & & $x$ & 1.5 \\
\hline & ZAC & $\mathbf{x}$ & & $\mathbf{x}$ & & & & & & $\mathbf{x}$ & $\mathbf{x}$ & & & $\mathbf{x}$ & 1.5 \\
\hline & VRU & \multicolumn{2}{|c|}{$\mathrm{V} 2 \mathrm{P}^{2}$} & $\mathbf{x}$ & & & & & $\mathbf{x}$ & $\mathbf{x}$ & $\mathbf{x}$ & & $\mathbf{x}$ & & 1.5 \\
\hline & $\mathrm{CCN}^{3}$ & $\mathbf{x}$ & & & & & & & & & & & & & 1.5 \\
\hline
\end{tabular}

Within infotainment tasks, the Traffic Information and Smart Routing service may also involve vehicle-to-vehicle (V2V) communication and decentralized environmental notification messages (DENMs). ${ }^{2}$ Vulnerable road user (VRU) services are based on vehicle-to-pedestrian (V2P) communication. ${ }^{3}$ No definitive description is given. Note: EBL, electronic brake light; EVA, emergency vehicle approaching; SSV, slow or stationary vehicle; TJW, traffic jam warning; RWW, road works warning; WTC, weather condition; CCRW, cooperative collision risk warning; MCA, motorcycle approaching; WWD, wrong way driving; VSGN, in-vehicle signage; VSPD, in-vehicle speed; PVD, probe vehicle data; SWD, shockwave damping; GLOSA, green light optimal speed advisory; SigV, signal violation/intersection safety; TSP, traffic signal priority; LZM, loading zone management; ZAC, zone access control; CCN, connected and cooperative navigation; V2I, vehicle-to-infrastructure; CAM, cooperative awareness message; SPaT, signal phase and timing; MAP, map data; IVI, in-vehicle information; ETSI, European Telecommunications Standards Institute; SC, safety critical; and nSC, nonsafety critical. 


\section{Current State of the Art of C-ITS Systems}

In this section, open-road and laboratory test activities for the development of cooperative driving expertise are outlined. Given the extensive nature of the topic addressed, what follows may not be considered exhaustive. Suffice it to think that connected driving accounts for around $5 \%$ of the total budget allocated for the societal challenge 'Smart Green and Integrated Transport' within the Horizon2020 research and innovation programme. As a result, there are several EU-funded projects that have been launched in the last years and many others will be initiated before the end of the seven year work programme. Instances of such projects are: AnaVANET [41], related to the development of a visualisation tool for vehicular networks; ENSEMBLE [42], related to track platooning services; MAVEN [43], related to intersection safety; 5GCar [44], related to fifth generation cellular communication; and AUTOPILOT [45], related to IoT V2X applications.

However, there are also several initiatives, such as testbeds developed by the academic community and research bodies, which, although not widely promoted or disclosed, turn out to be noteworthy and particularly relevant for taking a step forward in cooperative and automated driving tasks. First, in [46], the so-called IT-AV Automotive Environment, developed in Aveiro, is presented. It is a testbed comprising roadside units (RSUs), connected to the IT-AV datacentre through Ethernet links, and onboard units (OBUs), which are located in vehicles. They connect with each other via standard IEEE 802.11p/WAVE links and are connected to the RSUs and Internet through IEEE 802.11p/WAVE, IEEE $802.11 \mathrm{~g} / \mathrm{Wi}-\mathrm{Fi}$, or cellular links, thus enabling both V2V and V2I tasks. Therefore, on the one hand, OBUs can have access to vehicle information (such as position, heading, and speed), which are elaborated by the embedded in-car node processor to take local decisions and be notified to other vehicles. On the other hand, vehicles can also receive road information from the surroundings by means of embedded car video cameras and sensors. Such information will be transmitted to the OBUs using IEEE $802.11 \mathrm{~g} /$ Wi-Fi. A virtualized network function (VNF) video transcoding camera-based car overtaking scenario was evaluated as a use case. However, other VNFs can also be tested such as car crash detection, emergency info dissemination, and collision avoidance.

As shown in [47], in February 2018, Telefonica and Huawei presented the 5G-V2X testbed, developed in their 5G Joint Innovation Lab in Madrid, which adopted the latest 3GPP new radio (NR) standard. In particular, a novel, self-contained frame structure for radio transmission, based on the so-called ultra-reliable and low-latency communication (URLLC) mode, was implemented for V2X applications and tested in the case of vehicle platooning applications. Additionally, to further reduce transmission latency, sparse coded multiple access (SCMA)-based grant free access technology was also investigated.

Moreover, a modular framework was adopted for the testbed described in [48], whose effectiveness was exemplarily shown by implementing a crossing assistant scenario aimed at avoiding collisions at intersections. The testbed was based on seven different modules and a three-layer plug-in software architecture. The main components were: model cars at a scale of 1:18; a specifically developed camera-based positioning system; a central control unit responsible for management, car configuration, and car control; and a visualization unit providing the status of simulation on a graphical user interface (GUI) and showing which cars are communicating as well as what information they are exchanging. In particular, the model cars are equipped with a single board computer as a processing unit, an electronic brushed motor, special hardware for attaching different sensors, and a Wi-Fi communication unit.

The main advantage of the above organisation, based on different building blocks, is that each element is easy to manage and maintain since it is independent and self-contained. Indeed, a modular approach has similarly been adopted for the development of testbeds proposed in [49] and [50]. The former is based on a software-defined radio (SDR) platform and aims to satisfy the most stringent link-level communication requirements for $\mathrm{V} 2 \mathrm{X}$ applications, especially in the case of time-critical tasks such as emergency braking and cooperative emergency manoeuvres. The latter represents a communication-centric traffic light controller system based on the following major components: the 
RSU integrated with the smart traffic light manager (STLM) module, which is responsible for the creation and transmission of SPaT and MAP messages; the OBU with the human-machine interface (HMI) displaying information in a user-friendly way; the traffic light power manager (TLPM) module, which provides the required power for traffic lights and allows them to be controlled remotely; and the traffic management curve (TMC), which monitors and manages traffic flows, thus contributing to decreased traffic jams.

A testbed specifically developed for WTC applications was presented in [51]. It was devised by the Finnish Meteorological Institute (FMI)and Lapland UAS (Lapland University of Applied Sciences), and it was tailored for typical arctic winter conditions (i.e., snow and ice). In particular, the central element was represented by a combined road weather station (RWS)/RSU hotspot, which employed IEEE 802.11p communication as the primary channel. Therefore, vehicles exchanged data with the RSU during bypass, or whenever in communication range with it, and could further transmit such information to vehicles they met outside the catchment area of the RSU. However, if IEEE 802.11p communication was not available, vehicles could obtain information also by means of traditional Wi-Fi communication or, as a last resort, a 3G cellular link to obtain current data from the nearest RWS.

A testbed based on a system configuration specifically developed for vehicle platooning applications, instead, was described in [52]. It relied, exclusively, on V2V communication by means of $802.11 \mathrm{p}$ and 5G-V2X vehicular technologies without the use of sensor data. Although vehicle platooning applications generally envisage that both the leader and the followers send standardised messages, in this work the communication was considered unidirectional (i.e., it occurred only from the platoon leader to the follower). It was based on CAMs, which comprised header, payload, and platooning container, while the control algorithm adopted for calculation of desired acceleration and steering angle to follow the vehicle in front was based on a model predictive controller (MPC). Moreover, application of such a service in two urban scenarios, namely fast lane change and turning manoeuvres, was presented.

Additionally, [53] presented the WiSafeCar platform for testing accident and weather conditionrelated services. It was based on four main components (i.e., vehicles, the RSU, the linking point, and a mobile user) and relied on an intelligent hybrid wireless traffic safety network. IEEE 802.11p communication, together with a supporting $3 \mathrm{G}$ cellular network, was implemented. Moreover, the possibility of exploiting vehicle-based sensor information and observation data for providing real-time services was also investigated.

Finally, it is worth citing two works that provide considerable contribution to the development of vehicular networks. The first presented a real-world testbed called HarborNet [54], a vehicular mesh networking testbed that comprised OBUs installed in trucks, administration vehicles, tow boats, and patrol vessels; RSUs connected to the optic fibre backbone of the seaport; and cloud-based data and control systems. It allowed cloud-based code deployment, remote network control, and distributed data collection from moving vehicles, thus enabling a wide range of experiments and performance analyses. HarborNet operates at the Leixões seaport in Porto, Portugal; however, it can be easily tailored to support smart city services and cooperative driving applications. By contrast, the second work [55] presented a prototyping framework as guidance for the development of simulation environments aimed at testing cooperative advanced driver-assistance system (ADAS) applications, namely cooperative emergency lane change (CELC), cooperative adaptive cruise control (CACC), and parking autonomously in cooperative environments (PACE). The proposed approach was based on an iterative prototyping process of application refining and validation, which aimed at identifying a fair compromise between simulating multiple vehicles at the same time and accuracy in modelling their dynamics.

It is worth specifying that the above-mentioned testbeds are described mainly in functional terms, while relative technical specifications can be found in the references provided.

Obviously, also beyond Europe's borders, considerable attention is paid to cooperative and autonomous driving by public authorities, road operators, equipment suppliers, and research 
organisations. Indeed, pilot sites and test activities are often found in the United States [56-60], China [61-64], Canada [65,66], Australia [67,68], Singapore [69,70], and Japan [71,72]. An interesting difference is that, in some of these cases, testing is more focused on the urban environment, with some emblematic cases in which the pilot zone is so extensive as to be considered a fake town. Such cases represent mock contexts where experiments may be conducted in a supervised space; the first of its kind was M-city [73], established in 2015, which covers a 130,000 $\mathrm{m}^{2}$ area at the University of Michigan North Campus (Ann Arbor). It is a controlled simulation environment, specifically created for testing connected and autonomous driving. M-city test facilities consist, amongst other things, of various road surfaces, up to four-lane roads, roundabouts and tunnels, fixed and variable street lighting, as well as fixed and moveable buildings. Subsequently, in 2017, Uber established Almono [74], a mock city in the Hazelwood neighbourhood of Pittsburgh. It occupies 170,000 $\mathrm{m}^{2}$ and comprises a giant roundabout, fake cars, as well as roaming mannequins and containers meant to simulate, respectively, pedestrians and buildings. However, in late 2017, the opening of an even larger pilot zone, called K-city [75], was announced by the South Korean Ministry of Land, Infrastructure, and Transport. At the moment, only motorways are operational; however, once finished, K-city will occupy 320,000 $\mathrm{m}^{2}$. There will be several different driving conditions, including toll gates, pedestrian and train-track crossings, and even potholes and construction sites. Finally, it is worth mentioning the joint initiative of Google and its sister project Waymo (both controlled by Alphabet), aimed at testing autonomous vehicles in a fake city in California, called Castle [76], planned to reach an area of nearly $370,000 \mathrm{~m}^{2}$.

By contrast, within Europe's borders, to the best of the authors' knowledge, two fake urban environments are currently operational, namely Zala Zone [77] in Hungary and CERMcity [78] in Germany. The former is a test environment launched in 2017 and manged by the Automotive Proving Ground Zala LTD. It is planned to cover a total area of $250 \mathrm{ha}$, where it is possible to test both classic vehicle dynamics and cooperative driving functions as well as conduct validation testing for electric vehicles. CERMcity was built by RWTH Aachen University with funding from the Federal Ministry of Education and Research. It comprises intersections, straights, parking areas, pedestrian walkways and crossings, as well as a multifunctional area and, in terms of communication technology, adopts the latest cellular standards and multiple Wi-Fi connections.

\subsection{C-Roads Pilot Sites}

On completion of the picture, it is worth dedicating a special mention to the C-Roads Programme. It is a large-scale deployment programme on real-life equipped infrastructures across the EU, aimed at making progress in C-ITS expertise by following a learning-by-doing approach. In December 2017, C-Roads released an overview concerning pilot sites across the EU [79]. A brief description of each national case is provided below.

\subsubsection{Austria}

The Austrian C-Roads-Pilot builds on the core elements of the EU C-ITS Corridor project in Austria (ECo-AT-European Corridor Austrian Testbed) [80] and extends them to a motorway-based network. The services investigated are basically Day-1 applications and particular attention is paid to the ITS-G5 communication standard. The leading competent body is the Austrian motorway operator ASFINAG, which will cover a total of $300 \mathrm{~km}$ of motorways and will start cross-site tests during 2019. Finally, it is worth noting that Austria is one of the Member States involved in the European project C-ITS Corridor [81], together with Germany and The Netherlands.

\subsubsection{Germany}

C-Roads Germany comprises two pilot sites located, respectively, in Lower Saxony and Hessen, whose test activities are harmonised by the Federal Highway Research Institute (BASt). Specifically, the companies NORDSYS and OECON P\&S run the Lower Saxony pilot site, while the local public road operator Hessen Mobil is responsible for the second area. The tested services are the Day- 1 type, and 
both ITS-G5 and cellular communications are under study. According to the planned timeline, by the end of 2019, the entire ITS system in Lower Saxony with three C-ITS services, namely SSV, VSGN, and PVD, will be operational. On the other hand, in Hessen, PVD services are already operational and will be followed by RWW, SSV, and EVA applications throughout 2019. Finally, SWD, TJM, and GLOSA services will be operational in 2020. As stated above, Germany is one of the Member States involved in the European project C-ITS Corridor.

\subsubsection{The Netherlands}

The Dutch pilot area is situated in the south of the Netherlands. The tested applications are Day-1 type: RWW, VSGN, and GLOSA services will be implemented in a hybrid framework combining both ITS-G5 and cellular network technologies. Moreover, infotainment tasks and different use cases of the logistic service multimodal cargo transport optimization (MCTO) are planned to be tested.

The project coordinator is the Dutch Ministry of Infrastructure and Water Management, which has set up a test site project management team (TPMT) for handling test activities. The Provinces of Noord-Brabant and Utrecht are involved. The rolling-out phase is planned to last for the whole of 2019. Moreover, road shows will be performed in 2020.

Finally, the Netherlands plays a key role in two other European projects, namely C-ITS Corridor and InterCor-Interoperable Corridors [82]. Within the InterCor framework (in which the other Member States involved are Belgium, France, and the UK), two relevant demonstration events held in the Netherlands should be mentioned. The first was a testfest, which took place in July 2017 and involved RWW, VSGN, and PVD services. It was followed by the GLOSA Pre-Testfest in June 2018, aimed at evaluating the interoperability level reached in implementing GLOSA services.

\subsubsection{Belgium}

The Belgium case presents two different contexts to be analysed: Flanders and Wallonia. The Flemish pilot site involves all the motorways that form part of the core network in Flanders. The area concerned is the Belgium site falling in the InterCor deployment framework. In the Flemish pilot, only Day-1 services are investigated, while the communication technology analysed concerns the implementation of cellular networks that are tested in combination with the HERE Location Cloud and the local Traffic Management Centre (TMC). The aim is to develop a cloud-based 'virtual infrastructure' connecting road users with the TMC while allowing the TMC to directly interact with end users. The implementing bodies are: the Flemish Department of Mobility and Public Works, Tractebel Engineering SA, ITS Belgium, and HERE Technologies. In accordance with the declared timeline, all preparatory phases (i.e., use case definition, system setup, fine-tuning, and driver acquisition) have been completed. The next phases consist in carrying out preliminary test drives and making the whole system operational, which will perform trial activities for the whole of 2019. Finally, the ex-post evaluation will be carried out in 2020.

As regards Wallonia test activities, the services and communication technologies tested as well as the planned timeline are essentially the same as the Flemish case, except for the fact that ITS-G5 is also investigated in Wallonia. The partners involved are: SOFICO (technically assisted by the Public Service of Wallonia/Directorate of Road Traffic Management), Tractebel Engineering SA, and ITS Belgium.

\subsubsection{France}

C-Roads France builds on the results of the European project SCOOP@F [83]. The goal is to test cooperative services within two types of end-user services: services in the urban environment and at the urban/interurban interface as well as traffic information services increasing comfort on transit stretches. Among Day-1 services, the majority of functions are investigated with the exception of some signage applications, namely VSPD, SWD, SigV, and TSP. Moreover, the development of a C-ITS smartphone application is planned, supporting early I2V (Infrastructure-to-Vehicle) services such as logistic services as well as parking and park and ride information. As regards the communication 
technology implemented, it is represented by a hybrid framework, enabling a seamless switch between ITS-G5 and cellular for non-safety-critical applications.

The partners involved are public road operators (DIRs EST, Centre-Est, Atlantique, and Ouest), road operator concessionaries (Autoroutes Paris-Rhin-Rhône-APRR, Société des Autoroutes du Nord et de l'Est de la France-SANEF, and VINCI Autoroutes), car manufacturers (Renault, PSA group), research institutes (CEREMA, IFSTTAR, Telecom Paris Tech, Université d'Auvergne Clermont-Ferrand, and Université de Reims Champagne-Ardennes), security experts (IDnomic), mobility labs (Car2road, Transpolis), and the major urban nodes authorities (Strasbourg Eurométropole, Bordeaux Métropole). According to the timeline stated, the rolling-out phase has just started. Finally, it is worth pointing out that France, in turn, is one of the Member States involved in the European project InterCor.

\subsubsection{United Kingdom}

C-Roads UK pilot builds on the core elements of the A2/M2 CVC-A2/M2 Connected Vehicle Corridor project [84], which, in turn, represents the UK part of the InterCor programme. The beneficiary is the UK Department for Transport (DfT) and the partners involved are Highways England (HE), Transport for London (TfL), and Kent County Council (KCC). In particular, HE and KCC intend to deliver their services through both ITS-G5 and cellular networks in a hybrid framework, whilst Transport for London intends to deliver the services via cellular technologies alone. According to the planned timeline, the four services GLOSA, IVS, RWW, and PVD are currently operational, and the demonstration will be held until May 2019. It is worth mentioning the demonstration event Hybrid Testfest, held in October 2018 in the UK, with the collaboration of other InterCor partners that aims at testing the implementation of the above Day-1 services within a hybrid G5/cellular framework.

\subsubsection{Nordic Countries (Denmark, Sweden, Norway, and Finland)}

Nordic countries are described in a unique, comprehensive framework since they are part of a joint initiative, namely the NordicWay project [85], and C-Roads pilot sites are essentially physically overlapping with those involved in the NordicWay programme. Strictly speaking, other countries are also involved in joint initiatives outlined in formally recognised European projects; however, in such cases, the European project and C-Roads tests differ, thus justifying the choice of describing the countries involved separately.

In light of the above, Denmark, Sweden, Norway, and Finland have a twofold aim: i) connecting their traffic management centres and their backbone road system with the common NordicWay architecture and ii) testing C-ITS applications in use-cases that are particularly relevant to their own national context.

In Denmark, C-Roads test activities, run by the Danish Road Directorate, are fully included in the NordicWay programme [86].

Also for Sweden, the limit between the NordicWay programme and C-Roads pilot sites is very vague; however, in this case, some Day-1.5 services (i.e., traffic information and smart routing; connected and cooperative navigation) are also being analysed. Finally, a large number of public and private organisations are involved in test activity under the leadership of the Swedish Transport Administration. Amongst others, we find: Telefonaktiebolaget LM Ericsson, Volvo Car Corporation, Chalmers University of Technology, KTH-Integrated Transport Research Lab (ITRL), Combitech AB, Triona AB, RISE Interactive Institute AB, Kapsch TrafficCom AB, Mindconnect AB, IBM Svenska AB, Swarco Sverige AB, Technolution AB, and Springworks AB.

In Norwegian pilot sites, two main goals are pursued under the leadership of Norwegian Public Roads Administration. The first is to test Day- 1 and Day-1.5 services on peripheral networks, where rural routes have only poor cellular connectivity and no full access to main power. Moreover, connected and automated driving tasks on major freight routes are investigated. Finally, test activities within the Finnish pilot sites aim to investigate Day-1 and Day-1.5 services on the core network but also test automated driving in snowy and icy arctic conditions. In this case, under the leadership of the public 
authorities of the Finnish Transport Agency (FTA) and Finnish Transport Safety Agency (Trafi), studies are performed by three coalitions led by the Lapland University of Applied Sciences, Sensible 4 Ltd., and VTT Technical Research Centre of Finland.

Obviously, most of the deployment activities aim to achieve a high level of interoperability, among the Nordic countries themselves and with other European countries, by means of numerous cross-border tests. Indeed, as regards the communication technologies analysed, they are essentially cellular-based; however, for those services that can also be implemented with ITS-G5 standards, both technologies are tested in an interoperability perspective. Overall, the Nordic countries have covered all Day-1 applications, except for SWD services. Moreover, the Nordic case represents, among those presented, the first instance in which advanced C-ITS services (i.e., CCRW and CCN) are involved in testing activities. A specific description of C-ITS tasks investigated in every single country will be provided below. Finally, the above-mentioned pilot sites are planned to be operational for the whole of 2019.

\subsubsection{Portugal}

C-Roads Portugal involves the Atlantic Corridor in Portugal and covers major sections of the core and comprehensive networks, together with Lisbon and Porto urban nodes. In particular, the programme is developed through five pilot sites whose activities are specified below.

- Pilot 1 for designing a National Single Point of Access (SPA) prototype able to cover information for around $3390 \mathrm{~km}(20 \%)$ of the network and developing an SPA mobile application (SPApp) covering Day-1 services

- Pilot 2 for testing Day-1 and Day-1.5 on different kinds of roads (metropolitan areas, interurban roads, streets, and highways) using a hybrid G5/cellular communication system. Pilot activities cover over $460 \mathrm{~km}$ of the core and comprehensive network, including cross-border sections in Valença and Caia and roads giving access to urban nodes of Lisbon and Porto

- Pilot 3 for providing connected and autonomous vehicles with automation on levels two and three of the Trans-European Networks-Transport (TEN-T) network, also using a hybrid G5/cellular communication framework

- Pilot 4 consisting of the following subactivities focused on the Lisbon node: testing traffic monitoring and travel time prediction tasks by means of cellular technology; investigating infotainment services on parking availability with, in addition, the development of an in-vehicle app based on a hybrid communication framework; analysing bus corridor prioritisation services supported by cellular technologies; and evaluating potential benefits of the integration of private car usage with other transport modes in the last mile of interurban motorway corridors in a hybrid communication architecture

- Pilot 5 for investigating applications on traffic prediction services, by means of cellular and Wi-fi technologies, and testing the incorporation in the Porto network of a smart bus covering a corridor of around $1.4 \mathrm{~km}$, using the DATEXII communications protocol and cellular communication technologies

More than 30 partners are involved, including: Instituto da Mobilidade e dos Transportes, Universidade do Porto, Ascendi Grande Lisboa, Ascendi do Grande Porto, IP Telecom SA, Siemens SA, Vialivre SA, and GMVIS Skysoft SA. According to the planned timeline, by the end of 2020, C-ITS services will be implemented along $964 \mathrm{~km}$, ensuring a continuity of service in urban nodes and the core network. In all, 212 RSUs will be installed with 180 OBUs and 162 vehicles in operation. 
4.1.9. Spain

Similar to Portugal, C-Roads Spain develops through five pilot sites:

- $\quad$ DGT 3.0-located along the overall road network in Spain, with an extension of approximately $12,270 \mathrm{~km}$. It will be deployed by adopting cellular-based communication technologies (3G and $4 \mathrm{G} / \mathrm{LTE})$.

- $\quad$ SISCOGA Extended-including the extension of an existing test site infrastructure in the city of Vigo and its metropolitan area, which is already prepared to test ITS-G5 communication technology. It will cover $150 \mathrm{~km}$.

- Madrid Calle 30-located along the road 'Calle 30' in Madrid, approximately $32 \mathrm{~km}$ long. C-ITS services will be deployed by using a hybrid G5/cellular approach.

- Cantabrian pilot-deployed along approximately $75 \mathrm{~km}$ in northern Spain, by using hybrid communications.

- Mediterranean pilot—deployed along approximately $125 \mathrm{~km}$ at selected road sections in Catalonia and Andalucia using hybrid technologies

Such areas present a high level of heterogeneity, thus allowing a wide spectrum of use-cases to be tested involving almost all Day-1 applications, except for TJW services, and various Day-1.5 functions, namely VRU, CCN, and infotainment tasks. Moreover, cross-border tests with Portugal are also planned within the SISCOGA Extended pilot site.

A large number of partners are involved, including public authorities (Dirección General de Tráfico, Dirección General de Carreteras, and Madrid Calle 30 SA), associations (ITS Spain, MLC ITS EUSKADI), private companies (Transport Simulation Systems SL, Indra Systemas SA, Ingartek Consulting SL, and SenseFields SL), Universities (Universidad Politécnica de Madrid, Universitat Politècnica de Catalunya, and Universitat de Valencia) and research centres (Asociación Centro Tecnológico Ceit-IK4, CTAG). According to the planned timeline, the programme will be rolled out during 2019 in two phases, while 2020 will be devoted to evaluation.

\subsubsection{Czech Republic}

Czech Republic activities, within the C-Roads programme, are outlined in six different pilot sites, from deployment and test (DT)1 to DT6. The services tested cover almost all Day-1 applications, excluding only shockwave damping and GLOSA tasks, and focus particularly on public transport safety. Both ITS-G5 and cellular-based technologies are being investigated with the aim of developing a hybrid framework. Several implementing bodies among transport operators, research organisations, and equipment supplier companies are involved, namely Road and Motorway Directorate (RSD), Brněnské komunikace company, Správa Železniční Dopravní Cesty (SŽDC) railway operator, public transport companies of Ostrava and Plzeň, INTENS Corporation, AŽD Praha, O2, T-Mobile, Škoda Auto, and Czech Technical University.

According to schedule, pilot sites from DT1 to DT5 were completed in 2018, while DT6 has just been activated. Therefore, all DT will be operational throughout 2019, and 2020 will be dedicated to ex-post evaluation.

\subsubsection{Hungary}

C-Roads Hungary project is focused on testing Day-1 services, while a hybrid DSRC/cellular technology is planned to be developed in the near future. The beneficiary is the Ministry of National Development (MND), while the implementing body is the Hungarian Public Road Nonprofit PLC. Other partners planned to be involved are: Budapest University of Technology and Economics (BUTE), Budapest Public Road PLC, Automotive Proving Ground Zala LTD (APZ), and ITS Hungary Association. According to the declared timeline, the test phase started in the second half of 2018 and is planned to last until the end of 2020, with an upgrade of equipment scheduled by the end of 2019 . Finally, cross-border tests are also envisaged in 2020. 


\subsubsection{Italy}

C-ROADS Italy project is focused on the following use-cases: anticipated disengagement of vehicle L3 highway pilot, truck platooning, passenger cars highway chauffeur, and combined scenarios of trucks and passenger cars.

The coordinator is the Italian Ministry of Infrastructure and Transport, which, at the beginning of 2018, set up a monitoring body exclusively dedicated to cooperative driving tasks. Among the implementing bodies there are different organisations, including: motorway operators (i.e., Brenner Motorway SPA, Autovie Venete Motorway SPA, and CAV-Concessioni Autostradali Venete Motorway SPA), carmakers (Iveco, PSA Group, Renault, Volvo, and Scania), research bodies (the Polytechnic University of Milan and FCA Research Centre), communication and logistic industries (North Italy Communications SRL, Telecom Italia SPA, Azcom Technology SRL, and Codognotto SNC). According to the planned timeline, by the end of 2019, $50 \mathrm{~km}$ of the Brenner Motorway, $10 \mathrm{~km}$ of the Autovie Venete Motorway, and $10 \mathrm{~km}$ of the CAV Motorway will be operational. Moreover, cross-border tests with Austria are planned for 2020. Specifically, ITS-G5 and cellular communication technologies will be tested.

\subsubsection{Slovenia}

C-Roads Slovenia pilot sites have been developed in two phases. The first, in which both ITS-G5 and cellular networks are tested, is planned along $100 \mathrm{~km}$ of the TEN-T core network. In the second phase, planned to start in 2020, roadside C-ITS-G5 infrastructure will be extended to the pilot length of $300 \mathrm{~km}$, including critical points connected with the Central C-ITS-G5 Server real-time platform located at the Dragomelj Traffic Management Centre. The implementing bodies are the Ministry of Infrastructure and the Motorway Company of the Republic of Slovenia (DARS). The tested services are all Day-1 type, namely SSV, TJM, RWW, WTC, VSGN, VSPD, and GLOSA applications.

\subsubsection{Summary}

A synopsis of C-ITS services tested in each Member State within the C-Roads programme is shown in Table 2, giving a state-of-the-art overview of the Day-1 and Day-1.5 applications investigated.

Table 2. C-ITS services tested in C-Roads pilot sites.

\begin{tabular}{|c|c|c|c|c|c|c|c|c|c|c|c|c|c|c|c|c|c|c|c|c|c|c|}
\hline & & \multicolumn{21}{|c|}{ C-ITS Services } \\
\hline & & \multicolumn{13}{|c|}{ Day-1 } & \multicolumn{8}{|c|}{ Day-1.5 } \\
\hline & & $\begin{array}{l}\text { E } \\
\text { B } \\
\text { L }\end{array}$ & $\begin{array}{l}\text { E } \\
\text { V } \\
\text { A }\end{array}$ & $\begin{array}{c}\text { SS } \\
\text { V }\end{array}$ & $\begin{array}{c}\mathbf{T} \\
\mathbf{J} \\
\mathbf{W}\end{array}$ & $\begin{array}{l}\mathbf{R} \\
\mathbf{W} \\
\mathbf{W}\end{array}$ & $\begin{array}{l}\text { W } \\
\text { TC }\end{array}$ & $\begin{array}{l}\text { V } \\
S \\
G \\
N\end{array}$ & $\begin{array}{l}\text { V } \\
\text { S } \\
P \\
D\end{array}$ & $\begin{array}{l}\text { P } \\
\text { V } \\
\text { D }\end{array}$ & $\begin{array}{c}\text { S } \\
\text { W } \\
\text { D }\end{array}$ & $\begin{array}{l}\text { G } \\
\text { L } \\
\text { O } \\
\text { S } \\
\text { A }\end{array}$ & $\begin{array}{l}\mathrm{S} \\
\mathrm{i} \\
\mathrm{g} \\
\mathbf{V}\end{array}$ & $\begin{array}{c}\text { TS } \\
\text { P }\end{array}$ & Info & $\begin{array}{l}\mathbf{L} \\
\mathbf{Z} \\
\mathbf{M}\end{array}$ & $\begin{array}{l}\mathrm{Z} \\
\mathrm{A} \\
\mathrm{C}\end{array}$ & $\begin{array}{l}\mathbf{V} \\
\mathbf{R} \\
\mathrm{U}\end{array}$ & $\begin{array}{l}\mathrm{C} \\
\mathrm{C} \\
\mathrm{R} \\
\mathrm{W}\end{array}$ & $\begin{array}{l}\text { M } \\
\text { C } \\
\text { A }\end{array}$ & $\begin{array}{l}\text { W } \\
\text { W } \\
\text { D }\end{array}$ & $\begin{array}{l}\mathrm{C} \\
\mathrm{C} \\
\mathrm{N}\end{array}$ \\
\hline \multirow{3}{*}{ 品 } & Austria & & & $\checkmark$ & $\checkmark$ & $\checkmark$ & $\checkmark$ & $\checkmark$ & $\checkmark$ & $\checkmark$ & & & & & & & & & & & & \\
\hline & Germany & & $\checkmark$ & $\checkmark$ & $\checkmark$ & $\checkmark$ & & $\checkmark$ & & $\checkmark$ & $\checkmark$ & $\checkmark$ & & & & & & & & & & \\
\hline & The Netherlands & & & & & $\checkmark$ & & $\checkmark$ & & $\checkmark$ & & $\checkmark$ & & & $\checkmark$ & & & & & & & \\
\hline \multirow{3}{*}{ 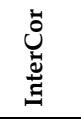 } & Belgium & & & $\checkmark$ & $\checkmark$ & $\checkmark$ & $\checkmark$ & & $\checkmark$ & & $\checkmark$ & & & & & & & & & & & \\
\hline & France & $\checkmark$ & $\checkmark$ & $\checkmark$ & $\checkmark$ & $\checkmark$ & $\checkmark$ & $\checkmark$ & & $\checkmark$ & & $\checkmark$ & & & $\checkmark$ & & & & & & & \\
\hline & United Kingdom & & & & & $\checkmark$ & & $\checkmark$ & & $\checkmark$ & & $\checkmark$ & & & & & & & & & & \\
\hline \multirow{10}{*}{ 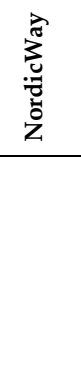 } & Denmark & & & $\checkmark$ & $\checkmark$ & $\checkmark$ & $\checkmark$ & & & $\checkmark$ & & & & & & & & & & & & \\
\hline & Finland & & $\checkmark$ & $\checkmark$ & & $\checkmark$ & $\checkmark$ & $\checkmark$ & $\checkmark$ & $\checkmark$ & & $\checkmark$ & $\checkmark$ & $\checkmark$ & $\checkmark$ & & & & & & & \\
\hline & Norway & $\checkmark$ & & $\checkmark$ & & $\checkmark$ & $\checkmark$ & $\checkmark$ & $\checkmark$ & $\checkmark$ & & $\checkmark$ & $\checkmark$ & & $\checkmark$ & & & & $\checkmark$ & & & \\
\hline & Sweden & $\checkmark$ & $\checkmark$ & & & $\checkmark$ & & $\checkmark$ & & & & $\checkmark$ & & $\checkmark$ & $\checkmark$ & & & & & & & $\checkmark$ \\
\hline & Portugal & $\checkmark$ & $\checkmark$ & $\checkmark$ & $\checkmark$ & $\checkmark$ & $\checkmark$ & $\checkmark$ & $\checkmark$ & $\checkmark$ & $\checkmark$ & $\checkmark$ & $\checkmark$ & $\checkmark$ & $\checkmark$ & & $\checkmark$ & & & & $\checkmark$ & $\checkmark$ \\
\hline & Spain & $\checkmark$ & $\checkmark$ & $\checkmark$ & & $\checkmark$ & $\checkmark$ & $\checkmark$ & $\checkmark$ & $\checkmark$ & $\checkmark$ & $\checkmark$ & $\checkmark$ & $\checkmark$ & $\checkmark$ & & & $\checkmark$ & & & & $\checkmark$ \\
\hline & Czech Republic & $\checkmark$ & $\checkmark$ & $\checkmark$ & $\checkmark$ & $\checkmark$ & $\checkmark$ & $\checkmark$ & $\checkmark$ & $\checkmark$ & & & $\checkmark$ & $\checkmark$ & & & & & & & & \\
\hline & Hungary & & & & $\checkmark$ & $\checkmark$ & $\checkmark$ & $\checkmark$ & $\checkmark$ & $\checkmark$ & & $\checkmark$ & $\checkmark$ & & & & & & & & & \\
\hline & Italy & $\checkmark$ & & $\checkmark$ & $\checkmark$ & $\checkmark$ & $\checkmark$ & $\checkmark$ & $\checkmark$ & $\checkmark$ & & & & & & & & & & & & \\
\hline & Slovenia & & & $\checkmark$ & $\checkmark$ & $\checkmark$ & $\checkmark$ & $\checkmark$ & $\checkmark$ & & & $\checkmark$ & & & & & & & & & & \\
\hline
\end{tabular}


In particular, as can be seen, Day-1 services are implemented in the majority of EU countries (16 over 28). Moreover, it is worth noting that, for VSGN, GLOSA, RWW, and other hazardous location notification applications, shared deployment principles and specifications are established for assuring interoperability across the Union [87]. Table 2 also shows that Day-1.5 services are less widely investigated since they are not undergoing tests at all in 9 out of 16 Member States, as highlighted by grey lines in the table; if infotainment tasks are excluded, the proportion drops to a quarter. Information in Table 2 could also be combined with those in Figure 2, which provides a synoptic view about where C-ITS service deployment activities are located across Europe and the magnitude of tests performed. In particular, black regions and lines have been adopted for indicating areas in which a greater number of C-ITS services is evaluated. Clearly, it represents only a drafted illustration, since the goal is to provide an at-a-glance view of the current situation. Moreover, it is worth specifying that only activities having an importance from a pan-European point of view have been considered, while initiatives of national importance (e.g., the Smart Roads project in Italy or the Human Drive initiative in UK) have been neglected. However, with all the previous in mind, it can be seen that the most intense test activity occurs on TEN-T corridors that, not surprisingly, have been included in formally recognised European projects such as C-ITS corridor and InterCor. In particular, the involvement in deployment activities (and possibly in the level of funding distribution) is very heterogeneous across different countries in the EC. Southern Europe and some parts of Eastern Europe are completely excluded, and some differences are very undeniable among involved states. For example, Portugal and Italy are in a totally different situation, with the former having all the most relevant infrastructures under development and the latter being involved in testing activities only for a small part of the country. Furthermore, within the NordicWay project, Sweden and Denmark are less proactive than other partners, and the latter is the only partner country in which exclusively Day-1 services are under test. 
Legenda:

$\otimes$ Hard activity test area $\bigcirc$ Soft activity test area $\quad$ Hard activity test corridor

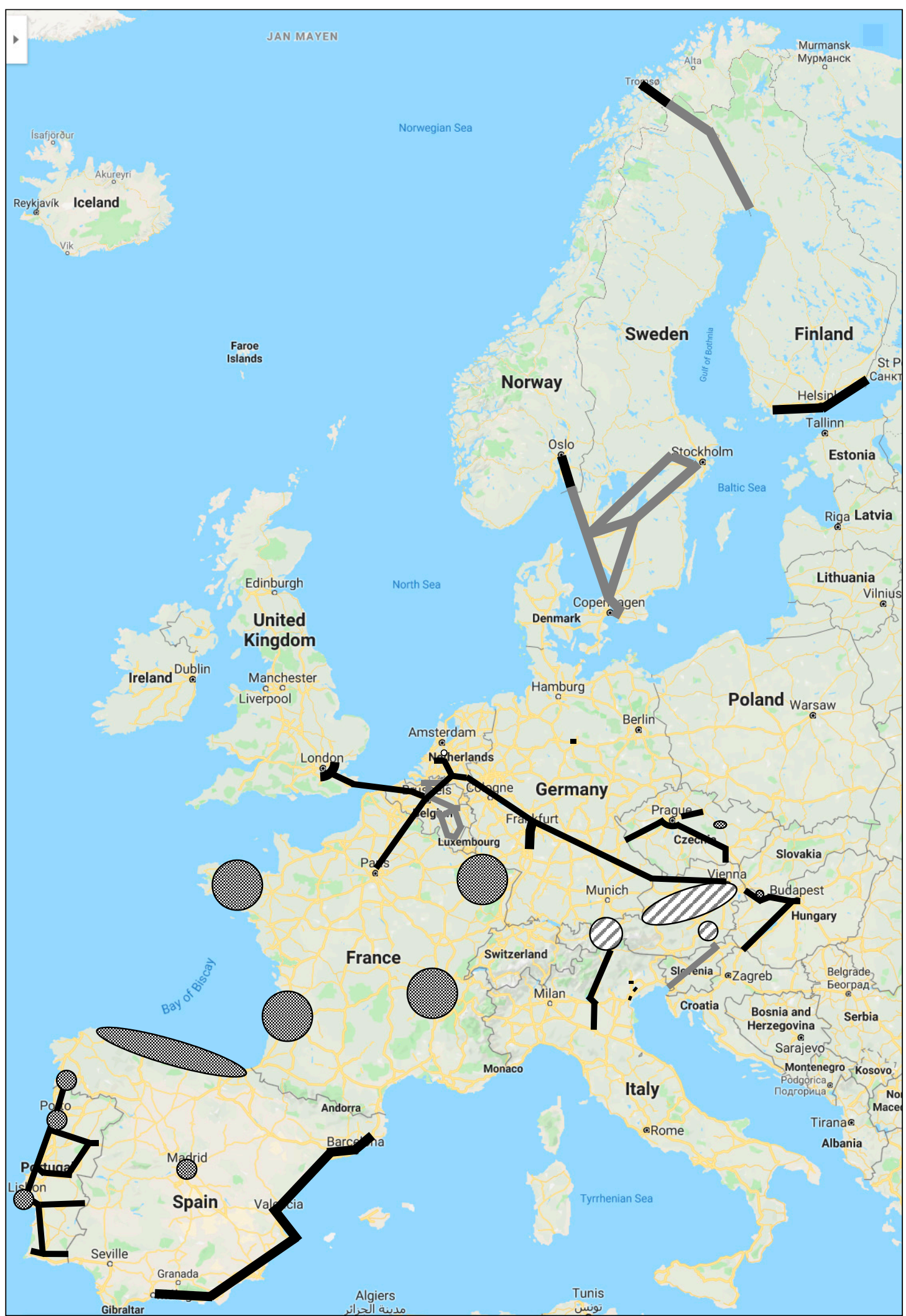

Figure 2. Synoptic view of C-ITS deployment activities across the EU. 
Regarding communication technologies, it is worth specifying that although ITS-G5 and cellular-based communications represent the most mature technologies, additional systems are being tested such as Wi-Fi and Bluetooth (in France, the Czech Republic, and Portugal), radio data (in Portugal), and digital audio broadcasting (in Germany).

Finally, analysing the types of stakeholders involved in the C-Roads programme [88], a considerable heterogeneity is observable across Member States. The number of entities involved is obviously consistent with the quantity of actions carried out, ranging from 1-2 (in the case of Austria and Slovenia) to over 30 (for Portugal and Sweden). The actual drivers of the development process in different countries are also quite heterogeneous. In fact, countries with a well-established tradition in the automotive sector (such as Germany, Sweden, and France) present considerable involvement of car-makers and equipment suppliers gravitating around the automobile industry. By contrast, countries like Austria, the Netherlands, the UK, and Denmark show a more centralised management behaviour, with public authorities being the main players. However, as can be seen, the most diverse organisations are involved, thus confirming the need to adopt a multidisciplinary approach for successful deployment of C-ITS applications.

\section{Concluding Remarks}

The above overview provides an insight into the current deployment activities in the European Union within the field of C-ITS and V2X communication. Best practices across the European Union were investigated, with the aim of outlining the state of play in early 2019, which was identified as the start time for deployment of mature services.

In sum, against a total budget of around EUR 300 million allocated for cooperative driving development by the EU's framework programme Horizon 2020, a resulting picture emerges with the following highlights. Regarding infrastructural pilot sites, a common trend can be identified. Indeed, generally from 2016 to 2018, preparatory phases were performed such as use-case definition, specification, development, and validation. Thus, they will be ready to start rolling out in 2019 at the latest and will be devoted to evaluation in 2020. The most mature aspect is the technological one, where ITS-G5 and cellular-based communications have been shown to be ready for implementation, possibly in different contexts and use-cases. Hybrid frameworks have been tested as well, allowing implementation of C-ITS services to potentially benefit from the advantages offered by both communication systems. By contrast, with reference to C-ITS services viewed from an applicative perspective involving final users/drivers, a fairly heterogeneous development can be noted; RWW appears as the only service investigated in almost all the testbeds, followed by PVD, SSV, and VSGN. We identified three possible reasons for such a different state of play among the above-mentioned C-ITS applications.

First, vehicle-side technological and applicative issues could delay the deployment of some tasks. In this regard, it may be stated that the use of cameras, radar transmitters, sensors, laser scanners, and digital maps appears consolidated. Moreover, several ADASs, such as adaptive cruise control, emergency braking, backup cameras, and self-parking systems, have already been installed on commercial vehicles. However, in order to take a step forward and enable more complex tasks, as shown by [89], systematisation and harmonisation of issues related to perception, localisation, and the decision-making process in a fail-safe scenario are required. Perception issues concern the development of suitable algorithms able to read the surrounding environment with a high degree of reliability in all operational domains, distinguishing, for instance, a stationary motorcycle from a bicyclist riding at the roadside. Essentially, object analysis is made complex by the various randomnesses involved (e.g., movement, background, and weather conditions), which account for the failure of most test activities and at times also lead to fatal crashes (even not in C-ITS testbeds). As regards the question of localisation, it is related to the error range of the GPS signal. In this regard, the EU undertook further development of Galileo services and their accuracy for driverless mobility. Finally, the human decision-making process should be suitably modelled in a software environment, thus reproducing driver choice behaviour. This is undoubtedly the most complex aspect to be addressed. 
The latter issue leads us to the second possible reason for the lack of development in certain C-ITS services, which is a methodological issue (i.e., the lack of a robust procedure for successfully finalising the task). This happens, for instance, in the case of advanced driving information tasks. Indeed, although the technology for acquiring and delivering information is mature, the extremely dynamic nature of such a task means that services concerning, for instance, traffic information and smart routing, even if increasingly popular, are not yet consolidated from the point of view of their ability to be predictive and pre-emptive. Indeed, after receiving certain information with traffic forecasting characteristics, users change their behaviour, thus altering the utility and reliability of the same information for the rest of the drivers [90]. There is no such complication, instead, when stationary objects are involved, such as the case of road works. Therefore, as shown by [91], the following research questions are still being debated: How do drivers change their behaviour because of warnings/information given by C-ITS services? Is safety affected by changes in driver behaviour because of C-ITS services?

The third reason is related to the spread of equipped C-ITS systems. Indeed, issues concerning the standardisation of information and procedures can be identified in the case of services involving a large number of vehicles or different traffic participants such as shockwave damping, vulnerable road user protection, and connected and cooperative navigation.

Our overview, albeit comprehensive, was not exhaustive: it mainly focused on roadside deployment activities. However, in the field of V2X communication, infrastructure equipment and implementation of in-vehicle technologies represent two sides of the same coin. They are complementary to one another, as demonstrated also by the $\mathrm{C} 2 \mathrm{C}$ roadmap framework, and need to be deployed synergistically. Nevertheless, if roadside deployment can be handled in a strategic perspective, with standardisation and regulation principles dictated from a centralised authority, the carmakers side will present a more fragmentary framework affected by particular aspects of each company, such as the know-how possessed and in-house policies adopted. Another limitation of the presented work concerns the initiatives mentioned and related stakeholders. Indeed, given the extent of the phenomenon as well as its ever-changing nature, it is next to impossible to provide a complete list of the countless deployment activities and public/private organisations involved. Therefore, far from any claim to be exhaustive, our contribution aims to provide a valid synopsis of the current state of affairs so as to steer future efforts along the right lines.

Finally, as shown by the study described in [92], when autonomous vehicles become available, a sizable increase occurs in vehicle miles travelled and number of trips. This is hardly surprising: it would be as if each user had a personal chauffeur. Such an event could negatively affect benefits in terms of sustainability and congestion reduction, of which mention is generally made. Hence, the authors suggest including in the evaluation step a study focusing on the above point, thus providing a realistic and reliable representation of what the future with autonomous vehicles would look like.

Author Contributions: Conceptualization, M.B., L.P. and G.N.B.; Methodology, L.D. and G.N.B., Investigation, M.B.; Data curation, M.B. and L.P.; Visualization, M.B. and L.P.; Writing-original draft preparation, M.B., L.P., L.D. and G.N.B.; Writing-review and editing, M.B., L.P., L.D. and G.N.B.; Supervision, L.P. L.D. and G.N.B.; Validation, L.D. and G.N.B.

Funding: This research was partially funded by Federico II University of Naples (Italy), grant number 000009-ALTRO_R-2016-LD "Models and technologies for transportation systems".

Conflicts of Interest: The authors declare no conflict of interest. The funders had no role in the design of the study; in the collection, analyses, or interpretation of data; in the writing of the manuscript, or in the decision to publish the results. 
Abbreviations

\begin{tabular}{|c|c|}
\hline $\mathrm{A} 2 / \mathrm{M} 2 \mathrm{CVC}$ & A2/M2 Connected Vehicle Corridor \\
\hline ADAS & Advanced Driver Assistance Systems \\
\hline AG & Amsterdam Group \\
\hline APZ & Automotive Proving ground Zala \\
\hline BASt & Federal Highway Research Institute \\
\hline BUTE & Budapest University of Technology and Economics \\
\hline $\mathrm{C} 2 \mathrm{C}-\mathrm{CC}$ & Car2Car Communication Consortium \\
\hline CACC & Cooperative Adaptive Cruise Control \\
\hline CAM & Cooperative Awareness Message \\
\hline CAV & Concessioni Autostradali Venete \\
\hline CCAM & Cooperative Connected and Automated Mobility \\
\hline $\mathrm{CCN}$ & Connected and Cooperative Navigation \\
\hline CCRW & Cooperative Collision Risk Warning \\
\hline CELC & Cooperative Emergency Lane Change \\
\hline C-ITS & Cooperative-Intelligent Transportation Systems \\
\hline CTAG & Centro Tecnológico de Automoción de Galicia \\
\hline DENM & Decentralized Environmental Notification Message \\
\hline DfT & Department for Transport \\
\hline DSRC & Dedicated Short-Range Communication \\
\hline DT & Deployment and Tests \\
\hline EBL & Emergency electronic Brake Light \\
\hline EC & European Commission \\
\hline ECo-AT & European Corridor Austrian Testbed \\
\hline ETSI & European Committee for Standardisation \\
\hline EU & European Union \\
\hline EVA & Emergency Vehicle Approaching \\
\hline FMI & Finnish Meteorological Institute \\
\hline FTA & Finnish Transport Agency \\
\hline GLOSA & Green Light Optimal Speed Advisory \\
\hline GUI & Graphical User Interface \\
\hline $\mathrm{HE}$ & Highways England \\
\hline HGV & Heavy Goods Vehicle \\
\hline HLG & High Level Group \\
\hline HLN & Hazardous Location Notifications \\
\hline HMI & Human Machine Interface \\
\hline $\mathrm{I} 2 \mathrm{~V}$ & Infrastructure-to-Vehicle \\
\hline ITRL & Integrated Transport Research Lab \\
\hline IVI & In-Vehicle Information \\
\hline KCC & Kent County Council \\
\hline LZM & Loading Zone Management \\
\hline MAC & Medium Access Control \\
\hline MAP & Map Data \\
\hline MCA & Motorcycle Approaching indication \\
\hline MCTO & Multimodal Cargo Transport Optimization \\
\hline MND & Ministry of National Development \\
\hline MPC & Model Predictive Controller \\
\hline NR & New Radio \\
\hline OBU & On-Board Unit \\
\hline OEMs & Original Equipment Manufacturers \\
\hline PACE & Parking Autonomously in Cooperative Environments \\
\hline PHY & Physical \\
\hline PVD & Probe Vehicle Data \\
\hline RSD & Road and Motorway Directorate \\
\hline RSU & Road-Side Unit \\
\hline
\end{tabular}




$\begin{array}{ll}\text { RWS } & \text { Road Weather Station } \\ \text { RWW } & \text { Road Works Warning } \\ \text { SAE } & \text { Society of Automotive Engineers } \\ \text { SCMA } & \text { Sparse Coded Multiple Access } \\ \text { SDR } & \text { Software Defined Radio } \\ \text { SigV } & \text { Signal Violation/ Intersection safety } \\ \text { SPA } & \text { Single Point of Access } \\ \text { SPApp } & \text { Single Point of Access Application } \\ \text { SPaT } & \text { Signal Phase and Timing } \\ \text { SSV } & \text { Slow or Stationary Vehicle } \\ \text { STLM } & \text { Smart Traffic Light Manager } \\ \text { SWD } & \text { Shockwave Damping } \\ \text { SŽDC } & \text { Správa Železniční Dopravní Cesty } \\ \text { TEN-T } & \text { Trans-European Networks - Transport } \\ \text { TfL } & \text { Transport for London } \\ \text { TJW } & \text { Traffic Jam ahead Warning } \\ \text { TLPM } & \text { Traffic Light Power Manager } \\ \text { TMC } & \text { Traffic Management Centre } \\ \text { TPMT } & \text { Test site Project Management Team } \\ \text { Trafi } & \text { Finnish Transport Safety Agency } \\ \text { TSP } & \text { Traffic Signal Priority } \\ \text { TTG } & \text { Time to Green } \\ \text { URLLC } & \text { Ultra-Reliable and Low-Latency Communication } \\ \text { V2I } & \text { Vehicle-to-Infrastructure } \\ \text { V2P } & \text { Vehicle-to-Pedestrian } \\ \text { V2V } & \text { Vehicle-to-Vehicle } \\ \text { V2X } & \text { Vehicle-to-Everything } \\ \text { VNF } & \text { Virtualized Network Function } \\ \text { VRU } & \text { Vulnerable Road User } \\ \text { VSGN } & \text { in-Vehicle Signage } \\ \text { VSPD } & \text { in-Vehicle Speed limits } \\ \text { WTC } & \text { Weather Conditions } \\ \text { WWD } & \text { Wrong Way Driving } \\ \text { ZAC } & \text { Zone Access Control } \\ & \end{array}$

\section{References}

1. Commission Delegated Regulation of 13.3.2019 Supplementing Directive 2010/40/EU of the European Parliament and of the Council with Regard to the Deployment and Operational Use of Cooperative Intelligent Transport Systems; European Commission: Brussels, Belgium, 2019; Available online: http://ec.europa.eu/transparency/regdoc/r ep/3/2019/EN/C-2019-1789-F1-EN-MAIN-PART-1.PDF (accessed on 30 April 2019).

2. European Commission, Cooperative, Connected and Automated Mobility (CCAM). Available online: ec.europa.eu/transport/themes/its/c-its_en (accessed on 30 April 2019).

3. Car2Car Communication Consortium (C2C-CC). Available online: www.car-2-car.org (accessed on 30 April 2019).

4. Amsterdam Group (AG). Available online: amsterdamgroup.mett.nl (accessed on 30 April 2019).

5. Botte, M.; Pariota, L.; D'Acierno, L.; Bifulco, G.N. C-ITS communication: An insight on the current research activities in the European Union. J. Transp. Syst. 2018, 3, 52-63.

6. Society of Automotive Engineers (SAE). Taxonomy and Definitions for Terms Related to Driving Automation Systems for On-Road Motor Vehicles. Standard SAE J3016-2018. 2018. Available online: https://www.sae.org/st andards/content/j3016_201806 (accessed on 30 April 2019).

7. On the Road to Automated Mobility: An EU Strategy for Mobility of the Future; European Commission: Brussels, Belgium, 2018; Available online: https:/ec.europa.eu/transport/sites/transport/files/3rd-mobility-pack/com20 180283_en.pdf (accessed on 30 April 2019). 
8. Ensuring That Europe Has The Most Competitive, Innovative and Sustainable Automotive Industry of the 2030s and Beyond. GEAR 2030 Final Report. 2017. Available online: https://ec.europa.eu/docsroom/documents/26081/at tachments/1/translations/en/renditions/pdf (accessed on 30 April 2019).

9. European Telecommunications Standards Institute (ETSI). Intelligent Transport Systems (ITS); Vehicular Communications; Basic Set of Applications; Definitions. Standard ETSI TR 102 638-1. 2009. Available online: www.etsi.org/deliver/etsi_tr/102600_102699/102638/01.01.01_60/tr_102638v010101p.pdf (accessed on 30 April 2019).

10. European Telecommunications Standards Institute (ETSI). Intelligent Transport Systems (ITS); Infrastructure to Vehicle Communication; Electric Vehicle Charging Spot Notification Specification. Standard ETSI TS 101 556-1. 2012. Available online: https://www.etsi.org/deliver/etsi_ts/101500_101599/10155601/01.01.01_60/ts_1015560 1v010101p.pdf (accessed on 30 April 2019).

11. International Organization for Standardization (ISO). Intelligent Transport Systems; Event-Based Probe Vehicle Data. Standard ISO/TS 29284. 2012. Available online: www.iso.org/obp/ui\#iso:std:iso:ts:29284:ed-1:v1:en (accessed on 30 April 2019).

12. European Telecommunications Standards Institute (ETSI). Intelligent Transport Systems (ITS); V2X Applications; Part 1: Road Hazard Signalling (RHS) Application Requirements Specification. Standard ETSI TS 101 539-1. 2013. Available online: www.etsi.org/deliver/etsi_ts/101500_101599/10153901/01.01.01_60/ts_10153901v010101p.pdf (accessed on 30 April 2019).

13. International Organization for Standardization (ISO). Intelligent Transport Systems; Forward Vehicle Collision Warning Systems; Performance Requirements and Test Procedures. Standard ISO 15623. 2013. Available online: www.iso.org/obp/ui/\#iso:std:iso:15623:ed-2:v1:en (accessed on 30 April 2019).

14. International Organization for Standardization (ISO). Intelligent Transport Systems (ITS); Cooperative Intersection Signal Information and Violation Warning Systems (CIWS); Performance Requirements and Test Procedures. Standard ISO 26684. 2015. Available online: www.iso.org/obp/ui\#tiso:std:iso:26684:ed-1:v1:en (accessed on 30 April 2019).

15. International Organization for Standardization (ISO). Intelligent Transport Systems; Cooperative Systems; Data Exchange Specification for In-Vehicle Presentation of External Road and Traffic Related Data. Standard ISO/TS 17425. 2016. Available online: www.iso.org/obp/ui/\#iso:std:iso:ts:17425:ed-1:v1:en (accessed on 30 April 2019).

16. European Telecommunications Standards Institute (ETSI). Intelligent Transport Systems (ITS); V2X Applications; Part 2: Intersection Collision Risk Warning (ICRW) Application Requirements Specification. Standard ETSI TS 101 539-2. 2018. Available online: www.etsi.org/deliver/etsi_ts/101500_101599/10153902/01.01.01_60/ts_1015390 2v010101p.pdf (accessed on 30 April 2019).

17. European Telecommunications Standards Institute (ETSI). Intelligent Transport Systems (ITS); Vehicular Communications; Basic Set of Applications; Part 2: Specification of Cooperative Awareness Basic Service. Standard ETSI EN 302 637-2. 2014. Available online: www.etsi.org/deliver/etsi_en/302600_302699/30263702/01.03.01_3 0/en_30263702v010301v.pdf (accessed on 30 April 2019).

18. European Telecommunications Standards Institute (ETSI). Intelligent Transport Systems (ITS); Vehicular Communications; Basic Set of Applications; Part 3: Specifications of Decentralized Environmental Notification Basic Service. Standard ETSI EN 302 637-3. 2014. Available online: www.etsi.org/deliver/etsi_en/302600_302699/3 0263703/01.02.01_30/en_30263703v010201v.pdf (accessed on 30 April 2019).

19. Santa, J.; Pereñíguez, F.; Moragón, A.; Skarmeta, A.F. Vehicle-to-Infrastructure messaging proposal based on CAM/DENM specifications. In Proceedings of the 6th IFIP/IEEE Wireless Days Conference-WDays 2013, Valencia, Spain, 13-15 November 2013. [CrossRef]

20. European Telecommunications Standards Institute (ETSI). Intelligent Transport Systems (ITS); Testing; Conformance Test Specifications for Signal Phase and Timing (SPAT) and Map (MAP) Part 1: Test Requirements and Protocol Implementation Conformance Statement (PICS) pro Forma. Standard ETSI TS 103 191-1. 2015. Available online: www.etsi.org/deliver/etsi_ts/103100_103199/10319101/01.01.01_60/ts_10319101v010101p.pdf (accessed on 30 April 2019).

21. European Telecommunications Standards Institute (ETSI). Intelligent Transport Systems (ITS); Testing; Conformance Test Specifications for Signal Phase And Timing (SPAT) and Map (MAP) Part 2: Test Suite Structure and Test Purposes (TSSETP). Standard ETSI TS 103 191-2. 2015. Available online: www.etsi.org/deliver/etsi_ts /103100_103199/10319102/01.01.01_60/ts_10319102v010101p.pdf (accessed on 30 April 2019). 
22. European Telecommunications Standards Institute (ETSI). Intelligent Transport Systems (ITS); Testing; Conformance test Specifications for Signal Phase and Timing (SPAT) and Map (MAP) Part 3: Abstract Test Suite (ATS) and Protocol Implementation eXtra Information for Testing (PIXIT). Standard ETSI TS 103 191-3. 2015. Available online: www.etsi.org/deliver/etsi_ts/103100_103199/10319103/01.01.01_60/ts_10319103v010101p.pdf (accessed on 30 April 2019).

23. International Organization for Standardization (ISO). Intelligent Transport Systems; Cooperative ITS; Dictionary of In-Vehicle Information (IVI) Data Structures. Standard ISO/TS 19321. 2015. Available online: www.iso.org/ obp/ui/\#iso:std:iso:ts:19321:ed-1:v1:en (accessed on 30 April 2019).

24. European Telecommunications Standards Institute (ETSI). Intelligent Transport Systems (ITS); Vehicular Communications; Basic Set of Applications; Facilities Layer Protocols and Communication Requirements for Infrastructure Services. Standard ETSI TS 103 301. 2016. Available online: https://www.etsi.org/deliver/etsi_ts /103300_103399/103301/01.01.01_60/ts_103301v010101p.pdf (accessed on 30 April 2019).

25. European Telecommunications Standards Institute (ETSI). Intelligent Transport Systems (ITS); European Profile Standard for the Physical and Medium Access Control Layer of Intelligent Transport Systems Operating in the $5 \mathrm{GHz}$ Frequency Band. Standard ETSI ES 202 663. 2012. Available online: www.etsi.org/deliver/etsi_es/202600_2026 99/202663/01.01.00_50/es_202663v010100m.pdf (accessed on 30 April 2019).

26. Institute of Electrical and Electronics Engineers (IEEE). Wireless LAN Medium Access Control (MAC) and Physical Layer (PHY) Specifications. Standard IEEE 802.11. 2007. Available online: https://www.iith.ac.in/ \{\}t br/teaching/docs/802.11-2007.pdf (accessed on 30 April 2019).

27. Institute of Electrical and Electronics Engineers (IEEE). Wireless LAN Medium Access Control (MAC) and Physical Layer (PHY) Specifications-Amendment 6: Wireless Access in Vehicular Environments. Standard IEEE 802.11p. 2010. Available online: www.ietf.org/mail-archive/web/its/current/pdfqf992dHy9x.pdf (accessed on 30 April 2019).

28. Sjöberg, K.; Andres, P.; Buburuzan, T.; Brakemeier, A. Cooperative Intelligent Transport Systems in Europe. IEEE Veh. Technol. Mag. 2017, 12, 89-97. [CrossRef]

29. Filippi, A.; Moerman, K.; Daalderop, G.; Alexander, P.D.; Schober, F.; Pfliegl, W. Ready to Roll: Why 802.11p Beats LTE and 5G for V2X. NXP Semiconductors, Cohda Wireless and Siemens White Paper. 2016. Available online: https://www.siemens.com/content/dam/webassetpool/mam/tag-siemens-com/smdb/mobility/road/ connected-mobility-solutions/documents/its-g5-ready-to-roll-en.pdf (accessed on 30 April 2019).

30. European Telecommunications Standards Institute (ETSI). LTE; Service Requirements for V2X Services (3GPP TS 22.185 Version 14.3.0 Release 14). Standard ETSI TS 122 185. 2017. Available online: www.etsi.org/deliver/e tsi_ts/122100_122199/122185/14.03.00_60/ts_122185v140300p.pdf (accessed on 30 April 2019).

31. European Telecommunications Standards Institute (ETSI). 5G; Service Requirements for Enhanced V2X Scenarios (3GPP TS 22.186 Version 15.3.0 Release 15). Standard ETSI TS 122 186. 2018. Available online: https: //www.etsi.org/deliver/etsi_ts/122100_122199/122186/15.03.00_60/ts_122186v150300p.pdf (accessed on 30 April 2019).

32. 5G Automotive Association. The Case for Cellular V2X for Safety and Cooperative Driving. White Paper. 2016. Available online: http://5gaa.org/wp-content/uploads/2017/10/5GAA-whitepaper-23-Nov-2016.pdf (accessed on 30 April 2019).

33. Flament, M. Path Towards 5G for the Automotive Sector. 5G Automotive Association Presentation. 2018. Available online: http://www.3gpp.org/ftp/Information/presentations/presentations_2018/2018_10_17_tokyo /presentations/2018_1017_3GPP\%20Summit_07_5GAA_FLAMENT.pdf (accessed on 30 April 2019).

34. Rebbeck, T.; Stewart, J.; Lacour, H.A.; Killeen, A.; McClure, D.; Dunoyer, A. The Cost-Benefit Analysis on Cellular Vehicle-to-Everything (C-V2X) Technology and Its Evolution to 5G-V2X. 5G Automotive Association Report. 2017. Available online: http://5gaa.org/wp-content/uploads/2017/12/Final-report-for-5GAA-on-cellu lar-V2X-socio-economic-benefits-051217_FINAL.pdf (accessed on 30 April 2019).

35. 5G Americas. Cellular V2X Communications Towards 5G. White Paper. 2018. Available online: http://www.5gamericas.org/files/9615/2096/4441/2018_5G_Americas_White_Paper_Cellular_V2X_Comm unications_Towards_5G__Final_for_Distribution.pdf (accessed on 30 April 2019).

36. Fallgren, M.; Dillinger, M.; Alonso-Zarate, J.; Boban, M.; Abbas, T.; Manolakis, K.; Mahmoodi, T.; Svensson, T.; Laya, A.; Vilalta, R. Fifth-generation technologies for the connected car: Capable systems for Vehicle-to-Anything communications. IEEE Veh. Technol. Mag. 2018, 13, 28-38. [CrossRef] 
37. Härri, J.; Brens, F. Challenges and Opportunities of WiFi-based V2X Communications. In Proceedings of the VDI Conference on Digital Infrastructure \& Automotive Mobility, Berlin, Germany, 5-6 July 2017.

38. Papathanassiou, A.T.; Khoryaev, A. Cellular V2X as the Essential Enabler of Superior Global Connected Transportation Services. IEEE 5 G Tech Focus 2017, 1. Available online: https://futurenetworks.iee.org/tech-f ocus/june-2017/cellular-v2x (accessed on 30 April 2019).

39. Turley, A.; Moerman, K.; Filippi, A.; Martinez, V. C-ITS: Three Observations on LTE-V2X and ETSI ITS-G5-A Comparison. NXP Semiconductors White Paper. 2018. Available online: https://www.nxp.com/docs/en/whitepaper/CITSCOMPWP.pdf (accessed on 30 April 2019).

40. C-ITS Platform Phase II Cooperative Intelligent Transport Systems Towards Cooperative, Connected and Automated Mobility. European Commission Report. 2017. Available online: https://ec.europa.eu/transport/sites/transpo rt/files/2017-09-c-its-platform-final-report.pdf (accessed on 30 April 2019).

41. AnaVAnet Project. Available online: http://anavanet.net/ (accessed on 30 April 2019).

42. ESEMBLE Project. Available online: http://platooningensemble.eu (accessed on 30 April 2019).

43. MAVEN Project. Available online: http://www.maven-its.eu (accessed on 30 April 2019).

44. 5Gcar Project. Available online: https://5gcar.eu (accessed on 30 April 2019).

45. AUTOPILOT Project. Available online: http://autopilot-project.eu (accessed on 30 April 2019).

46. 5GINFIRE: IT-AV Automotive Environment. Available online: https://5ginfire.eu/it-av-automotive-testbed/ (accessed on 30 April 2019).

47. Telefonica and Huawei: 5G-V2X Testbed. EuropaWire, 2018. Available online: https://news.europawire.eu/telefonic a-and-huawei-complete-joint-5g-v2x-poc-test-in-their-5g-joint-innovation-lab-at-madrid-53202031254/eu-pressrelease/2018/02/08/ (accessed on 30 April 2019).

48. Hecker, T.; Zech, J.; Schäufele, B.; Gräfe, R.; Radusch, I. Model car testbed for development of V2X applications. J. Commun. 2011, 6, 115-124. [CrossRef]

49. Cao, H.; Gangakhedkar, S.; Ramadam Ali, A.; Gharba, M.; Eichinger, J. A testbed for experimenting 5G-V2X requiring Ultra Reliability and Low-Latency. In Proceedings of the 21st International ITG Workshop on Smart Antennas-WSA 2017, Berlin, Germany, 15-17 March 2017.

50. Varga, N.; Bokor, L.; Takacs, A.; Kovacs, J.; Virag, L. An architecture proposal for V2X communication-centric traffic light controller systems. In Proceedings of the 15th International Conference on ITS TelecommunicationsITST 2017, Warsaw, Poland, 29-31 May 2017.

51. Sukuvaara, T.; Mäenpää, K.; Ylitalo, R.; Konttaniemi, H.; Petäjäjärvi, J.; Veskoniemi, J.; Autioniemi, M. Vehicular networking road weather information system tailored for arctic winter conditions. Int. J. Commun. Netw. Inf. Secur. 2015, 7, 60-68.

52. Jain, V.; Lapoehn, S.; Frankiewicz, T.; Hesse, T.; Gharba, M.; Gangakhedkar, S.; Ganesan, K.; Hanwen, C.; Eichinger, J.; Ramadam Ali, A.; et al. Prediction based framework for Vehicle Platooning using Vehicular Communications. In Proceedings of the 2017 IEEE Vehicular Networking Conference-VNC 2017, Turin, Italy, 27-29 November 2017. [CrossRef]

53. Sukuvaara, T.; Ylitalo, R.; Katz, M. IEEE 802.11p Based Vehicular Networking Operational Pilot Field Measurement. IEEE J. Sel. Areas Commun. 2013, 31, 409-417. [CrossRef]

54. Ameixieira, C.; Cardote, A.; Neves, F.; Meireles, R.; Sargento, S.; Coelho, L.; Afonso, J.; Areias, B.; Mota, E.; Costa, R.; et al. HarborNet: A real-world testbed for vehicular networks. IEEE Commun. Mag. 2014, 52, 108-114. [CrossRef]

55. Massow, K.; Radusch, I. A rapid prototyping environment for Cooperative Advanced Driver Assistance Systems. J. Adv. Transport. 2018. [CrossRef]

56. Thompson, R. All-in-One Urban Mapping Using V2X Communication; University of Tennessee: Chattanooga, TN, USA, 2012; Available online: http://tsite.org/wp-content/uploads/2012/11/Presentation-3-\%E2\%80\%93All-In-One-Urban-Mapping-Using-V2x-Communication.pdf (accessed on 30 April 2019).

57. Lewis, B. V2X Test Beds for Faster, Cleaner, Safer Transportation. IoT Design, 2016. Available online: http:// iotdesign.embedded-computing.com/articles/v2x-test-beds-for-faster-cleaner-safer-transportation (accessed on 30 April 2019).

58. Nissan, Savari and UC Berkeley Make V2X Testbed in Sunnyvale. Telematics Wire, 2016. Available online: https://www.telematicswire.net/vehicle-telematics-vehicle-information-technology-and-navigatio n/nissan-savari-and-uc-berkeley-make-v2x-testbed-in-sunnyvale/ (accessed on 30 April 2019). 
59. Connected and Automated Vehicle Program Plan. Report of Virginia Department of Transportation. 2017. Available online: https://www.hrtpo.org/uploads/docs/061218\%20A3\%20-\%20Release_Final_VDOT_CAV_ Program_Plan_Fall_2017.pdf (accessed on 30 April 2019).

60. Evans, R. Transportation Research Center Begins Construction of New CAV Test Facility in Ohio. Automotive Testing Technology Online Magazine. 2018. Available online: https: //www.automotivetestingtechnologyinternational.com/videos/transportation-research-center-begin s-construction-of-new-cav-test-facility-in-ohio.html (accessed on 30 April 2019).

61. Spirent, Tata Elxsi V2X Automotive Test System Adopted by Researchers. GPS World, 2016. Available online: http://gpsworld.com/spirent-tata-elxsi-v2x-automotive-test-system-adopted-by-researchers (accessed on 30 April 2019).

62. NI and Shanghai University Collaborate on a 5G Ultra-Reliable Low-Latency Testbed for V2X Communications. National Instruments Press Release. 2018. Available online: http://www.ni.com/newsroom/release/ni-and-sha nghai-university-collaborate-a-5g-ultra-reliable-low-latency-testbed-for-v2x-communications/en (accessed on 30 April 2019).

63. Vella, H. Guangzhou Becomes Test-Bed for Self-Driving Cars. Techwire Asia. 2018. Available online: https://techwireasia.com/2018/02/guangzhou-becomes-test-bed-self-driving-cars (accessed on 30 April 2019).

64. Ying, W. Shanghai Takes Another Step to Push Connected Car Road Tests. China Daily. 2018. Available online: https://www.chinadailyhk.com/articles/238/62/107/1519963996724.html (accessed on 30 April 2019).

65. AUtomotive Testbed for Reconfigurable and Optimized Radio Access (AURORA). Available online: http: //rsl.ece.ubc.ca/aurora (accessed on 30 April 2019).

66. Ottawa Launches Canada's First on-Street Test of an Autonomous Vehicle. City of Ottawa Press Release. 2017. Available online: https://ottawa.ca/en/news/ottawa-launches-canadas-first-street-test-autonomous-vehicle (accessed on 30 April 2019).

67. All Aboard the Future of Sustainable Transport. Monash University Press Release. 2018. Available online: https://www.monash.edu/engineering/about-us/news-events/latest-news/articles/2018/all-aboard-t he-future-of-sustainable-transport (accessed on 30 April 2019).

68. Emery, K. Driverless Car to Hit Perth Roads in 2019. The West Australian. 2018. Available online: https://hewest.com.au/news/perth/driverless-car-to-hit-perth-roads-in-2019-ng-b88957514z (accessed on 30 April 2019).

69. Smart Mobility Program. Available online: http://www.infinitus.eee.ntu.edu.sg/Programmes/SMP/Pages/h ome.aspx (accessed on 30 April 2019).

70. Lakrintis, A. Singapore Emerges as Autonomous Vehicles Test Bed. Strategy Analytics, 2016. Available online: https://www.strategyanalytics.com/strategy-analytics/blogs/automotive/autonomous-vehicles/au tonomous-vehicles/2016/08/15/singapore-emerges-as-autonomous-vehicles-test-bed (accessed on 30 April 2019).

71. Hayashi, Y.; Memezawa, I.; Kantou, T.; Ohashi, S.; Takayama, K. Evaluation of Connected Vehicle Technology for Concept Proposal Using V2X Testbed. SEI Tech. Rev. 2017, 85, 10-14.

72. Action Plan for Realizing Automated Driving; Japanese Ministry of Economy, Trade and Industry Report; 2018. Available online: http://www.meti.go.jp/english/policy/mono_info_service/connected_industries/pdf/ad_v2 .0_hokokusho.pdf (accessed on 30 April 2019).

73. M-City. Available online: mcity.umich.edu (accessed on 30 April 2019).

74. Goldberg, M. Uber Built a Miniature Fake City in Pittsburgh to Test Self-Driving Cars. The Drive, 2017. Available online: http://www.thedrive.com/tech/15241/uber-built-a-miniature-fake-city-in-pittsburgh-to-te st-self-driving-cars (accessed on 30 April 2019).

75. Korea Partially Opens Test Bed Road for Autonomous Vehicles. The Korea Herald: Yonhap News. 2017. Available online: http://www.koreaherald.com/view.php?ud=20171106000459 (accessed on 30 April 2019).

76. Madrigal, A.C. Inside Waymo's Secret World for Training Self-Driving Cars. The Atlantic. 2017. Available online: https://www.theatlantic.com/technology/archive/2017/08/inside-waymos-secret-testing-and-simu lation-facilities/537648/ (accessed on 30 April 2019).

77. Zala Zone Project. Available online: https://zalazone.hu/en (accessed on 30 April 2019).

78. CERMcity. Available online: https://www.atc-aldenhoven.de/en/new-urban-environment.html (accessed on 30 April 2019). 
79. Detailed Pilot Overview Report. C-Roads Platform Report. 2017. Available online: https://www.c-roads.eu/filea dmin/user_upload/media/Dokumente/Detailed_pilot_overview_report_v1.0.pdf (accessed on 30 April 2019).

80. Eco-At Project. Available online: http://eco-at.info/ (accessed on 30 April 2019).

81. Cooperative ITS Corridor Project. Available online: http://c-its-korridor.de/?menuId=1\&sp=en (accessed on 30 April 2019).

82. InterCor Project. Available online: http://intercor-project.eu (accessed on 30 April 2019).

83. SCOOP@F Project. Available online: http://www.scoop.developpement-durable.gouv.fr/-en (accessed on 30 April 2019).

84. InterCor UK Section: A2/M2 Connected Vehicle Corridor (A2/M2 CVC). Available online: http://intercor-pro ject.eu/homepage/operations-united-kingdom (accessed on 30 April 2019).

85. NordicWay Project. Available online: http://vejdirektoratet.dk/EN/roadsector/Nordicway/NordicWay1/Page s/Default.aspx (accessed on 30 April 2019).

86. Olsen, E. NordicWay2. Report. 2017. Available online: http://vejdirektoratet.dk/EN/roadsector/Nordicway/D ocuments/D2.\%20NordicWay2_Presentation_v2.pdf (accessed on 30 April 2019).

87. Harmonised C-ITS Specifications for Europe. C-Roads Platform Report. 2018. Available online: https: //www.c-roads.eu/fileadmin/user_upload/media/Dokumente/Harmonised_specs_text.pdf (accessed on 30 April 2019).

88. C-Roads Pilots: Core Members. Available online: https://www.c-roads.eu/pilots/core-members.html (accessed on 30 May 2019).

89. Heineke, K.; Kampshoff, P.; Mkrtchyan, A.; Shao, E. Self-Driving Car Technology: When Will the Robots Hit the Road? McKinsey \& Company, 2018. Available online: https://www.mckinsey.com/industries/automotive -and-assembly/our-insights/self-driving-car-technology-when-will-the-robots-hit-the-road (accessed on 30 April 2019).

90. Ben-Elia, E.; Di Pace, R.; Bifulco, G.N.; Shiftan, Y. The impact of travel information accuracy on route-choice. Transp. Res. Part C Emerg. Technol. 2013, 26, 146-159. [CrossRef]

91. Evaluation and Assessment Plan. C-Roads Platform Report. 2018. Available online: https://www.c-roads.eu/f ileadmin/user_upload/media/Dokumente/C-Roads_WG3_Evaluation_and_Assessment_Plan_Final.pdf (accessed on 30 April 2019).

92. Harb, M.; Xiao, Y.; Circella, G.; Mokhtarian, P.L.; Walker, J.L. Projecting travelers into a world of self-driving vehicles: Estimating travel behavior implications via a naturalistic experiment. In Proceedings of the 97th Annual Meeting of the Transportation Research Board, Washington, DC, USA, 7-11 January 2018. 